\title{
Effect of Magnetohydrodynamic on Thin Films of Unsteady Micropolar Fluid through a Porous Medium
}

\author{
Gamal M. Abdel-Rahman \\ Department of Mathematics, Faculty of Science, Benha University, Benha, Egypt \\ E-mail:gamalm60@yahoo.com \\ Received July 9, 2011; revised August 22, 2011; accepted September 3, 2011
}

\begin{abstract}
In This paper, we deal with the study of the effect of magnetohydrodynamic on thin films of unsteady micropolar fluid through a porous medium. These Thin films are considered for three different geometries. The governing continuity, momentum and angular momentum equations are converted into a system of non-linear ordinary differential equations by means of similarity transformation. The resulting system of coupled non-linear ordinary differential equations is solved numerically by using shooting method. A representative set of numerical results in the three thin film flow problems for velocity and micro-rotation profiles are discussed and presented graphically. A comprehensive parametric study is carried out to show the effects of the micropolar fluid parameters, magnetic field parameter, permeability parameter and etc. on the obtained solutions.
\end{abstract}

Keywords: Unsteady Flow, MHD, Thin Film, Porous Medium, Micropolar Fluid

\section{Introduction}

Nowadays, the world has a great interest in the study of non-Newtonian fluids from both fundamental and practical point of view. The understanding of physics involved in the flows of such fluids can have immediate effects on polymer processing, coating, ink-jet printing, micro fluidics, geological flows in the earth mantle, homodynamic, the flow of colloidal suspensions, liquid crystals, additive suspensions, animal blood, turbulent shear flows and many others. In view of this, a lot of interest has been shown towards the study of non-Newtonian flows and hence extensive literature regarding analytic and numerical solutions is available on the topic. It is also accepted now that in general, the governing equations of non-Newtonian fluids are highly non-linear and of higher order than the Navier-Stokes equations. Because of the non-linearity and the inapplicability of the superposition principle, the exact solutions are even difficult to be obtained for the case of viscous fluids. Such exact solutions further narrow down when nonNewtonian fluids are taken into account. A lot of work has been carried out regarding the analytic solutions for flows of non-Newtonian fluids in cases where the involved equations have been linearized and in cases where the partial differential equations have been re- duced into ordinary differential equations. Some recent attempts in this direction have been made in the studies [1-10].

Due to complexity of fluids there is not a single constitutive equation which can describe the properties of all non-Newtonian fluids. In view of this, several nonNewtonian fluid models have been proposed.

In micropolar fluids, rigid particles contained in a small volume element and rotate about the center of the volume element are described by the micro-rotation vector. This local rotation of the particles is in addition to the usual rigid body motion of the entire volume element.

In micropolar fluid theory, the laws of classical continuum mechanics are augmented with additional equations that account for conservation of microinertia moments and balance of first stress moments that arise due to consideration of the microstructure in a material. Amongst these, a micropolar fluid model is introduced by Eringen [11-13]. This model includes the effects of local rotary and couple stresses. Physically, some fluids with additives, nemotogenic and smectogenic liquid crystals, flow of colloidal fluids, suspension solutions, blood, fluid with bar like elements may be represented by the mathematical model underlying micropolar fluids. The study of micropolar fluid mechanics has attracted 
the attention of many researchers. A good list of references on the published papers for this fluid can be found in Eringen [14] and Ishak et al. [15]. Recently, Lok et al. [16] analyzed the boundary layer flow of a micropolar fluid near the forward stagnation point of a plane surface. Nazar et al. [17] studied the stagnation point flow of a micropolar fluid towards a stretching sheet. The problem of the porous stretching sheet has been of great use in engineering studies. Numerical study for micropolar flow over a stretching sheet is presented by Moncef [18].

The work on the thin film flows of non-Newtonian fluids under various configurations is relatively of recent origin. Siddiqui et al. [19] discussed the thin film flows of a third grade fluid down an inclined plane. In [20] they examined the thin film flows of Sisko and Oldroyd-6 constant fluids on a moving belt. The thin film flow of a fourth grade fluid down a vertical cylinder is also analyzed by Siddiqui et al. [21]. Sajid et al. [22] studied the above mentioned on exact solutions for thin film flows of a micropolar fluid in the absence of a magnetic field, i.e $M=0$. The studied effect of MHD on thin films of a micropolar fluid in the absence of a porous medium, i.e $S=0$ by Abdel-Rahman [23].

Hence, the objective of the present paper is the study of the effect of magnetohydrodynamic on thin films of unsteady micropolar fluid through a porous medium. These Thin films are considered for three different geometries. A representative set of numerical results in the three thin film flow problems for velocity and microrotation profiles are discussed and presented graphically. A comprehensive parametric study is carried out to show the effects of the micropolar fluid parameters $\left(K, m_{1}, m_{2}\right.$ and $m_{3}$ ), magnetic field parameter, permeability parameter and etc., which are also discussed.

\section{Mathematical Analysis}

The equation of continuity and the conservation equations of linear momentum and angular momentum for an incompressible unsteady micropolar fluid, in the presence of magnetohydrodynamic through a porous medium, by neglecting the body force and body couple, are:

$$
\begin{gathered}
\nabla \cdot \boldsymbol{V}=0, \\
\rho \frac{D \boldsymbol{V}}{D t}=-\nabla p+(\mu+\kappa) \nabla^{2} \boldsymbol{V}+\kappa \nabla x \boldsymbol{\Omega} \\
+\boldsymbol{J} \times \boldsymbol{B}-\left(\mu / \rho k^{*}\right) \boldsymbol{V}, \\
\rho j \frac{D \boldsymbol{\Omega}}{D t}=(\alpha+\beta+\gamma) \nabla(\nabla \cdot \boldsymbol{\Omega}) \\
-\gamma \nabla x(\nabla x \boldsymbol{\Omega})+\kappa \nabla x V-2 \kappa \boldsymbol{\Omega} .
\end{gathered}
$$

Subject to appropriate initial and boundary conditions.
Here $\boldsymbol{V}, \boldsymbol{\Omega}$ and $\boldsymbol{B}$ represent the velocity, micro-rotation and total magnetic vectors respectively, $\rho$ and $j$ denote the density and the gyration parameters of the fluid. $p$ is the pressure and $\mu, \kappa, \alpha, \beta, k^{*}$ and $\gamma$ are the material constants. If the velocity, micro-rotation and magnetic components are $(u, v, 0),(0,0, N)$ and $\left(0,0, B_{0}\right)$ respectively, where $\boldsymbol{B}$ is the total magnetic field, so $\boldsymbol{B}=B_{0}+\boldsymbol{b}, \boldsymbol{b}$ is the induced magnetic field.

In the low magnetic Reynolds number approximations, in which the induced magnetic field $\boldsymbol{b}$ can be ignored, the magnetic body force becomes; (see e.g. [24])

$$
\boldsymbol{J} x \boldsymbol{B}=-\sigma B_{0}^{2} \boldsymbol{V}
$$

In which $\sigma$ is the electrical conductivity of the fluid.

Consider the two-dimensional flow of unsteady, incompressible and micropolar fluid in absence of pressure gradient [25] and applying the magnetic field $B_{0}$ perpendicular to the velocity field through a porous medium. Under the usual boundary layer approximation, the governing equations for this problem can be written as following:

Continuity equation:

$$
\frac{\partial \bar{u}}{\partial \bar{x}}+\frac{\partial \bar{v}}{\partial \bar{y}}=0
$$

Momentum equation in the $\bar{x}$-direction is:

$$
\begin{aligned}
\frac{\partial \bar{u}}{\partial t}+\bar{u} \frac{\partial \bar{u}}{\partial \bar{x}}+\bar{v} \frac{\partial \bar{v}}{\partial \bar{y}}= & F_{1}+\left(v+\frac{\kappa}{\rho}\right)\left[\frac{\partial^{2} \bar{u}}{\partial \bar{x}^{2}}+\frac{\partial^{2} \bar{u}}{\partial \bar{y}^{2}}\right] \\
& +\frac{\kappa}{\rho} \frac{\partial \bar{N}}{\partial \bar{y}}-\frac{\sigma B_{0}^{2}}{\rho} \bar{u}-\frac{\mu}{\rho k^{*}} \bar{u},
\end{aligned}
$$

Momentum equation in the $\bar{y}$-direction is:

$$
\begin{aligned}
\frac{\partial \bar{v}}{\partial t}+\bar{u} \frac{\partial \bar{v}}{\partial \bar{x}}+\bar{v} \frac{\partial \bar{v}}{\partial \bar{y}}= & F_{2}+\left(v+\frac{\kappa}{\rho}\right)\left[\frac{\partial^{2} \bar{v}}{\partial \bar{x}^{2}}+\frac{\partial^{2} \bar{v}}{\partial \bar{y}^{2}}\right] \\
& +\frac{\kappa}{\rho} \frac{\partial \bar{N}}{\partial \bar{x}}-\frac{\sigma B_{0}^{2}}{\rho} \bar{v}-\frac{\mu}{\rho k^{*}} \bar{v},
\end{aligned}
$$

Angular momentum equation:

$$
\begin{aligned}
\frac{\partial \bar{N}}{\partial t}+\bar{u} \frac{\partial \bar{N}}{\partial \bar{x}}+\bar{v} \frac{\partial \bar{N}}{\partial \bar{y}}= & \frac{\gamma}{\rho j}\left(\frac{\partial^{2} \bar{N}}{\partial \bar{x}^{2}}+\frac{\partial^{2} \bar{N}}{\partial \bar{y}^{2}}\right) \\
& -\frac{\kappa}{\rho j}\left(2 \bar{N}-\frac{\partial \bar{v}}{\partial \bar{x}}+\frac{\partial \bar{u}}{\partial \bar{y}}\right) .
\end{aligned}
$$

where, $\bar{u}$ and $\bar{v}$ are the respective velocity components in $\bar{x}$ - and $\bar{y}$ - directions, $\bar{N}$ is the micro- rotation or angular velocity whose direction of rotation in the $x y$ - plane, $v$ is the kinematics viscosity and $j, \gamma$ and $k$ are the micro inertial per unit mass, spin gradient viscosity and vortex viscosity, respectively. Here $\gamma$ is assumed to be given by [26]; 


$$
\gamma=(\mu+\kappa / 2) j
$$

In which $\mu$ is the dynamic viscosity and $j$ is the reference length. As pointed out by Ahmadi [27].

Equation (9) is invoked to allow Equations (5)-(8) to predict the correct behavior in the limiting case when microstructure effects become negligible and in this case micro-rotation is reduced to the angular velocity. The micro-rotation $N$ at the wall is related to the shear stress at the wall by the relation:

$$
N_{\omega}=-n \tau_{\omega}
$$

Where $N_{\omega}$ and $\tau_{\omega}$ are micro-rotation and shear stress at the wall and $n$ is a constant $(0 \leq n \leq 1)$.

The case at $n=0$ indicates that $N_{\omega}=0$, which represents concentrated particle flows, in which the microelements, close to the wall surface are unable to rotate [28]. This case corresponds to the strong concentration of microelements [29]. The case at $n=0.5$ indicates the vanishing of the anti symmetric part of the stress tensor and denotes weak concentration of microelements [27]. We now consider the thin film flows for both the cases.

\section{Thin Film Flow down an Inclined Plane}

In the presence of a magnetic field $\boldsymbol{B}$ through a porous medium, we consider the thin film of an incompressible micropolar fluid down an inclined plane. The ambient air is assumed stationary so that the flow is caused by gravity only. Also, the surface tension is assumed negligible and the film thickness $\delta$ is uniform, as shown in Figure 1. The velocity $\boldsymbol{V}$, microrotation $\boldsymbol{N}$ and the magnetic body force $\boldsymbol{J} \boldsymbol{x} \boldsymbol{B}$ are:

$$
\begin{aligned}
& \overline{\boldsymbol{V}}=[\bar{u}(\bar{y}), 0,0], \overline{\boldsymbol{N}}=[0,0, \bar{N}(\bar{y})], \\
& F_{1}=g_{1} \sin \alpha \text { and } \boldsymbol{J} x \boldsymbol{B}=-\sigma B_{0}^{2}[\bar{u}(\bar{y}), 0,0]
\end{aligned}
$$

In view of above definition of velocity, Equations (5) and (7) are satisfied automatically and Equations (6) and (8) give:

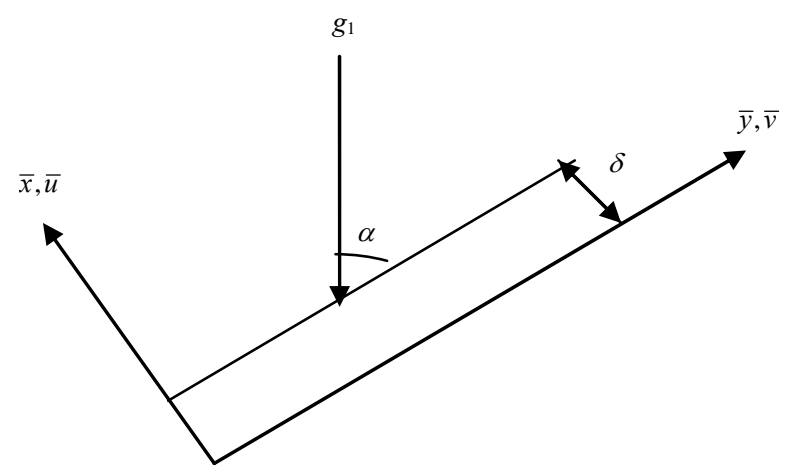

Figure 1. The geometry of the problem.

$$
\begin{gathered}
\left(v+\frac{\kappa}{\rho}\right) \frac{d^{2} \bar{u}}{d \bar{y}^{2}}+\frac{\kappa}{\rho} \frac{d \bar{N}}{d \bar{y}}+g_{1} \sin \alpha-\frac{\sigma B_{0}^{2}}{\rho} \bar{u}-\frac{\mu}{\rho k^{*}} \bar{u}=0 \\
\left(v+\frac{\kappa}{2 \rho}\right) \frac{d^{2} \bar{N}}{d \bar{y}^{2}}-\frac{\kappa}{\rho j}\left(2 \bar{N}+\frac{d \bar{u}}{d \bar{y}}\right)=0
\end{gathered}
$$

Subject to the boundary conditions

$$
\begin{aligned}
& \bar{u}=0, \bar{N}=-n \frac{d \bar{u}}{d \bar{y}} \text { at } \bar{y}=0 \\
& \frac{d \bar{u}}{d \bar{y}}=\bar{N}=0 \text { at } \bar{y}=\delta
\end{aligned}
$$

where $g_{1}$ is the gravity.

We introduce the following dimensionless variables:

$$
y=\frac{\bar{y}}{\delta}, u=\frac{\delta}{v} \bar{u} \text { and } N=\frac{\delta^{2}}{v} \bar{N} .
$$

where $j$ is equal to $\delta^{2}$. With the help of Equations (12)(14), we have:

$$
\begin{gathered}
(1+K) u^{\prime \prime}+K N^{\prime}+m_{1}-M u-S u=0, \\
\left(1+\frac{K}{2}\right) N^{\prime \prime}-K\left(2 N+u^{\prime}\right)=0,
\end{gathered}
$$

where $m_{1}=g_{1} \sin \alpha \delta^{3} / v^{2}$ is the parameter of film thickness $\delta, K=\kappa / \mu$ is the parameter of micropolar fluid, $M=\sigma B_{0}^{2} \delta^{2} / \mu$ is the magnetic parameter and $S=v / \delta^{2} k^{*}$ is the permeability parameter.

The boundary conditions (14) become:

$$
\begin{aligned}
& u(0)=0, N(0)=-n u^{\prime}(0) \\
& u^{\prime}(1)=N(1)=0
\end{aligned}
$$

\section{Thin Film Flow on a Moving Belt}

We consider presence of container with wide moving belt passing through it, and contain thin films of a micropolar fluid through a porous medium affected by magnetohydrodynamic. A the container. The belt moves in the vertical direction with velocity $U_{0}$ as shown in Figure 2. The belt picks up a thin film thickness $\delta$. The fluid is drained down due to gravity. We assume a steady laminar flow with uniform film thickness. The $x$ - axis is taken normal to the belt and therefore the velocity $\boldsymbol{V}$, micro-rotation $\boldsymbol{N}$ and the magnetic body force $\boldsymbol{J} x \boldsymbol{B}$ are:

$$
\begin{aligned}
& \overline{\boldsymbol{V}}=[0, \bar{v}(x), 0], \overline{\boldsymbol{N}}=[\bar{N}(x), 0,0], \\
& F_{2}=g_{1} \text { and } \boldsymbol{J} x \boldsymbol{B}=-\sigma B_{0}^{2}[0, \bar{v}(x), 0]
\end{aligned}
$$

Note that Equations (5) and (6) are identically satisfied while Equations (7) and (8) give: 


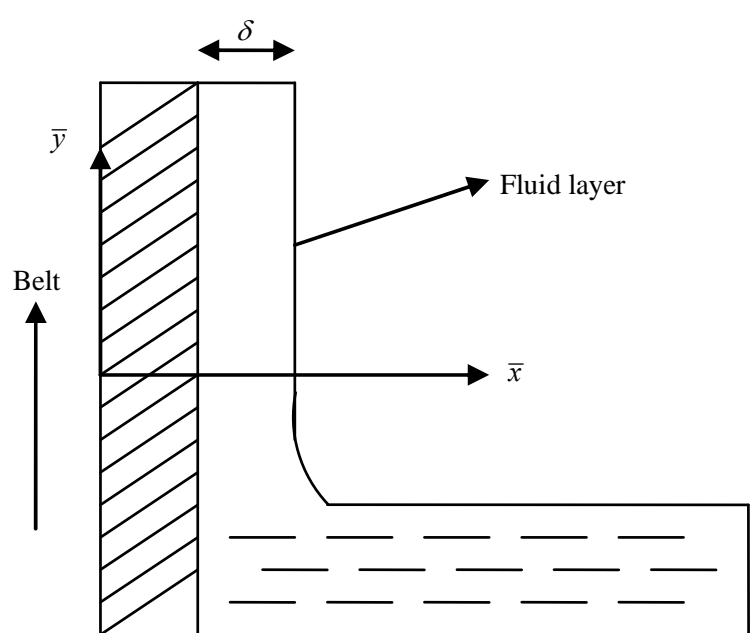

Figure 2. Geometry of the flow of moving belt through a micropolar fluid.

$$
\begin{gathered}
\left(v+\frac{\kappa}{\rho}\right) \frac{d^{2} \bar{v}}{d \bar{x}^{2}}-\frac{\kappa}{\rho} \frac{d \bar{N}}{d \bar{x}}-g_{1}-\frac{\sigma B_{0}^{2}}{\rho} \bar{v}-\frac{\mu}{\rho k^{*}} \bar{v}=0, \\
\left(v+\frac{\kappa}{2 \rho}\right) \frac{d^{2} \bar{N}}{d \bar{x}^{2}}-\frac{\kappa}{\rho j}\left(2 \bar{N}-\frac{d \bar{v}}{d \bar{x}}\right)=0 .
\end{gathered}
$$

For the problem under consideration, the boundary conditions are:

$$
\begin{aligned}
& \bar{v}=U_{0}, \bar{N}=-n \frac{d \bar{v}}{d \bar{x}} \text { at } \bar{x}=0 \\
& \frac{d \bar{v}}{d \bar{x}}=\bar{N}=0 \text { at } \bar{x}=\delta
\end{aligned}
$$

We introduce the following dimensionless variables:

$$
x=\frac{\bar{x}}{\delta}, v=\frac{\bar{v}}{U_{0}} \text { and } N=\frac{\delta}{U_{0}} \bar{N} .
$$

Equations (19)-(21) are reduced to:

$$
\begin{gathered}
(1+K) v^{\prime \prime}-K N^{\prime}-m_{2}-M v-S v=0, \\
\left(1+\frac{K}{2}\right) N^{\prime \prime}-K\left(2 N-v^{\prime}\right)=0 .
\end{gathered}
$$

where $m_{2}=g_{1} \delta^{2} / v U_{0}$ is the parameter of film thickness $\delta$,

The boundary conditions (21) become:

$$
\begin{aligned}
& v(0)=1, N(0)=-n v^{\prime}(0) \\
& v^{\prime}(1)=N(1)=0
\end{aligned}
$$

\section{Thin Film Flow down a Vertical Cylinder}

The governing equations here are [29]

$$
\begin{gathered}
\frac{\partial u}{\partial r}+\frac{u}{r}+\frac{\partial w}{\partial z}=0, \\
\frac{\partial u}{\partial t}+u \frac{\partial u}{\partial r}+w \frac{\partial u}{\partial z} \\
=F_{1}+\left(v+\frac{\kappa}{\rho}\right)\left[\frac{\partial^{2} u}{\partial r^{2}}+\frac{1}{r} \frac{\partial u}{\partial r}-\frac{u}{r^{2}}+\frac{\partial^{2} u}{\partial z^{2}}\right]-\frac{\kappa}{\rho} \frac{\partial N}{\partial z}, \\
\frac{\partial w}{\partial t}+u \frac{\partial w}{\partial r}+w \frac{\partial w}{\partial z}=F_{2}+\left(v+\frac{\kappa}{\rho}\right)\left[\frac{\partial^{2} w}{\partial r^{2}}+\frac{1}{r} \frac{\partial w}{\partial r}+\frac{\partial^{2} w}{\partial z^{2}}\right] \\
+\frac{\kappa}{\rho}\left(\frac{\partial N}{\partial r}+\frac{N}{r}\right)-\frac{\sigma B_{0}^{2}}{\rho} w-\frac{\mu}{\rho k^{*}} w
\end{gathered}
$$

$$
\begin{aligned}
\frac{\partial N}{\partial t}+u \frac{\partial N}{\partial r}+w \frac{\partial N}{\partial z}= & \frac{\gamma}{\rho j}\left[\frac{\partial^{2} N}{\partial r^{2}}+\frac{1}{r} \frac{\partial N}{\partial r}-\frac{N}{r^{2}}+\frac{\partial^{2} N}{\partial z^{2}}\right] \\
& -\frac{\kappa}{\rho j}\left(2 N+\frac{\partial w}{\partial r}-\frac{\partial u}{\partial z}\right) .
\end{aligned}
$$

where $u$ and $w$ are the velocity components in the $r-$ and $z$ - directions.

Consider a micropolar fluid falling on the outside surface of an infinitely long vertical cylinder of radius $R$ in the presence of a magnetic field $\boldsymbol{B}$ and a porous medium as shown in Figure 3. We also assume that, the uniform magnetic field of intensity $B_{0}$ acts in the radial direction and the effect of the induced magnetic field is negligible, which is valid when the magnetic Reynolds number is small.

The flow is in the form of a thin, uniform axisymmetric film of thickness $\delta$, in contact with stationary air. The velocity and micro-rotation are

$$
\boldsymbol{V}=[0,0, w(r)], \boldsymbol{N}=N(r) \text { and } F_{2}=g_{1}
$$

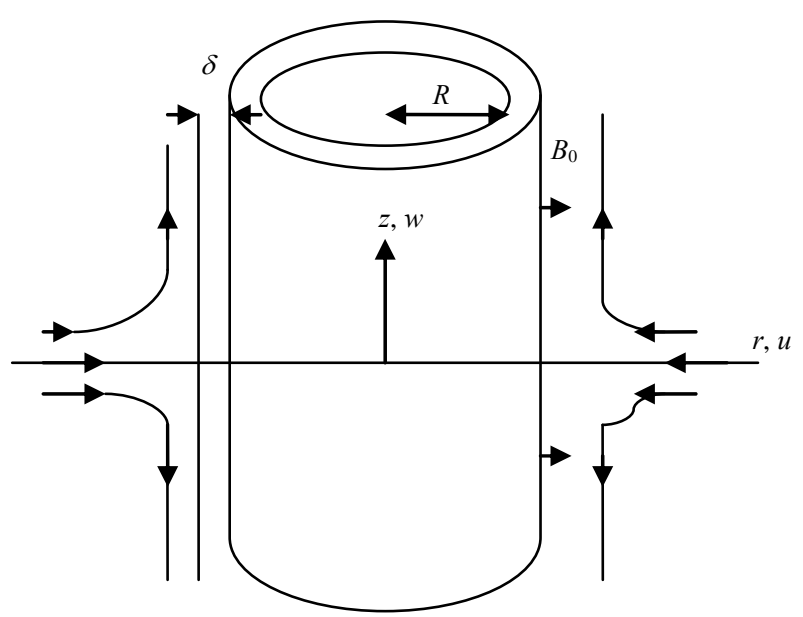

Figure 3. Physical model and coordinate system. 
Obviously, Equations (25) and (26) are satisfied identically and Equations (27) and (28) become:

$$
\begin{aligned}
& \left(v+\frac{\kappa}{\rho}\right)\left[\frac{d^{2} w}{d r^{2}}+\frac{1}{r} \frac{d w}{d r}\right]+\frac{\kappa}{\rho}\left(\frac{d N}{d r}+\frac{N}{r}\right) \\
& +g_{1}-\frac{\sigma B_{0}^{2}}{\rho} w-\frac{\mu}{\rho k^{*}} w=0, \\
& \frac{\gamma}{\rho j}\left[\frac{d^{2} N}{d r^{2}}+\frac{1}{r} \frac{d N}{d r}-\frac{N}{r^{2}}\right]-\frac{\kappa}{\rho j}\left(2 N+\frac{d w}{d r}\right)=0 .
\end{aligned}
$$

With the appropriate boundary conditions are:

$$
\begin{aligned}
& w=0, N=-n \frac{d w}{d r} \text { at } r=R \\
& \frac{d w}{d r}=N=0 \text { at } r=R+\delta
\end{aligned}
$$

Defining

$$
r=R \eta, w=\frac{v}{R} f \text { and } N=\frac{v}{R^{2}} \varphi
$$

Equations (29)-(31) are read as:

$$
\begin{gathered}
(1+K)\left(\eta f^{\prime \prime}+f^{\prime}\right)+K\left(\eta \varphi^{\prime}+\varphi\right) \\
+m_{3} \eta-M \eta f-S \eta f=0 \\
\left(1+\frac{K}{2}\right)\left(\eta^{2} \varphi^{\prime \prime}+\eta \varphi^{\prime}-\varphi\right)-K \eta^{2}\left(2 \varphi+f^{\prime}\right)=0
\end{gathered}
$$

where $m_{3}=g_{1} R^{3} / v^{3}$ is the parameter of film thickness $\delta$, $M=\sigma B_{0}^{2} R^{2} / \mu$ is the magnetic parameter and $S=v R^{2} / k^{*}$ is the permeability parameter.

The boundary conditions (31) become:

$$
\begin{aligned}
& f(1)=0, \varphi(1)=-n f^{\prime}(1) \\
& f^{\prime}(d)=\varphi(d)=0 .
\end{aligned}
$$

where $d=1+\frac{\delta}{R}$.

\section{Numerical Results and Discussions}

The system of the non-linear ordinary differential equations together with the boundary conditions are solved numerically by using Shooting method. Numerical results are presented for velocity and micro-rotation fields in the three thin film flow problems of a micropolar fluid: 1) flow down an inclined plane, 2) flow on a moving belt and 3) flow down a vertical cylinder, with the boundary layer for different parameters of the problem including micropolar fluid parameters $\left(K, m_{1}, m_{2}\right.$ and $\left.m_{3}\right)$ and the magnetic parameter for all ( $n=0.0$ and $n=0.5)$.
For the three considered problems, Figures $\mathbf{4}$ and $\mathbf{5}$ show magnetic field effect on a) the velocity and b) the micro-rotation profiles, it is noted that, the increase of the magnetic parameter $\mathrm{M}$ leads to; at $n=0.0$ and $n=0.5$, the velocity decreases and at $n=0.0$, the micro-rotation decreases in two cases 1) and 3) but it increases in case 2) while at $n=0.5$ the micro-rotation decreases.

Figures 6 and 7 show micropolar fluid parameter $K$ effect on a) the velocity and b) the micro-rotation profiles, it is noted that, the increase of the micropolar fluid parameter $K$ leads to; at $n=0.0$ and $n=0.5$ the velocity decreases in two cases 1 ) and 3 ) but it increases in case 2) and at $n=0.0$, the micro-rotation decreases while at $n=$ 0.5 it decreases in two cases 1 ) and 3) but it increases in case 2). However, the velocity and the micro-rotation profiles are greater for $n=1 / 2$ when compared with the case at $n=0.0$. We have also prepared Figures 8 and 9 just to see the effects of film thickness $\delta$ on a) the velocity and b) the micro-rotation profiles. It is seen that, the increase of the film thickness $\delta$ results in; at $n=0.0$ and $n=0.5$, the velocity increases in two cases 1 ) and 3) but it decreases in case 2) and at $n=0.0$, the micro-rotation increases in two cases 1) and 2) but it decreases in case 3 ), while at $n=0.5$, the micro-rotation increases in two cases 1) and 3) but it decreases in case 2). Furthermore, this increase is enhanced when the value of $n$ increases from zero to $1 / 2$.

Figures 10 and 11 show porous medium effect on a) the velocity and b) the micro-rotation profiles, it is noted that, the increase of the porous medium parameter $S$ leads to; at $n=0.0$ and $n=0.5$, the velocity decreases while at $n=0.0$ the micro-rotation decreases and at $n=$ 0.5 the micro-rotation decreases in two cases 1 ) and 3) but it increases in case 2).

\section{Conclusions}

In this paper, we have studied numerically the effect of magnetohydrodynamic on thin films of unsteady micropolar fluid through a porous medium. These Thin films are considered for three different geometries, named: 1) flow down an inclined plane, 2) flow on a moving belt and 3) flow down a vertical cylinder. From the present study we have found that:

Numerical results are presented for velocity and micro-rotation fields in the three thin film flow problems of a micropolar fluid. The results are graphically presented and the influence of micropolar fluid parameters, the porous medium parameter and the magnetic parameter are discussed for strong and weak concentrations of the microelements. It is observed that, the rotation of the microelements at the boundary increases the velocity 

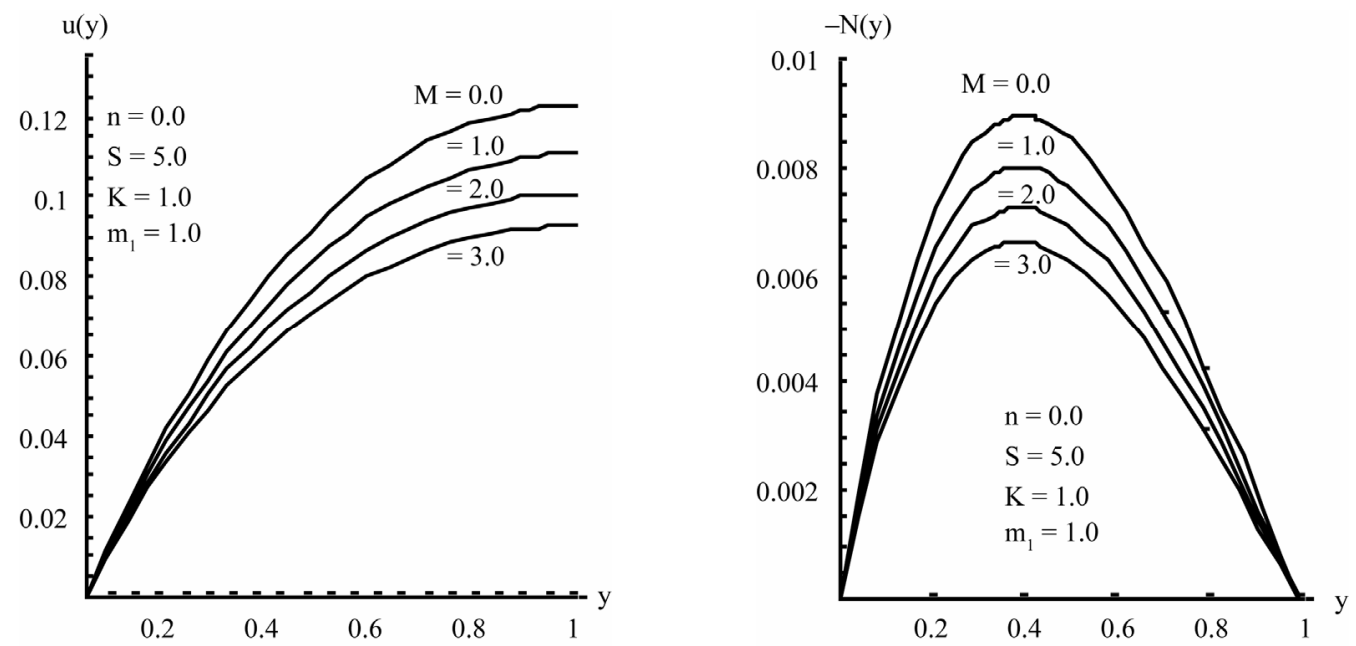

(1)
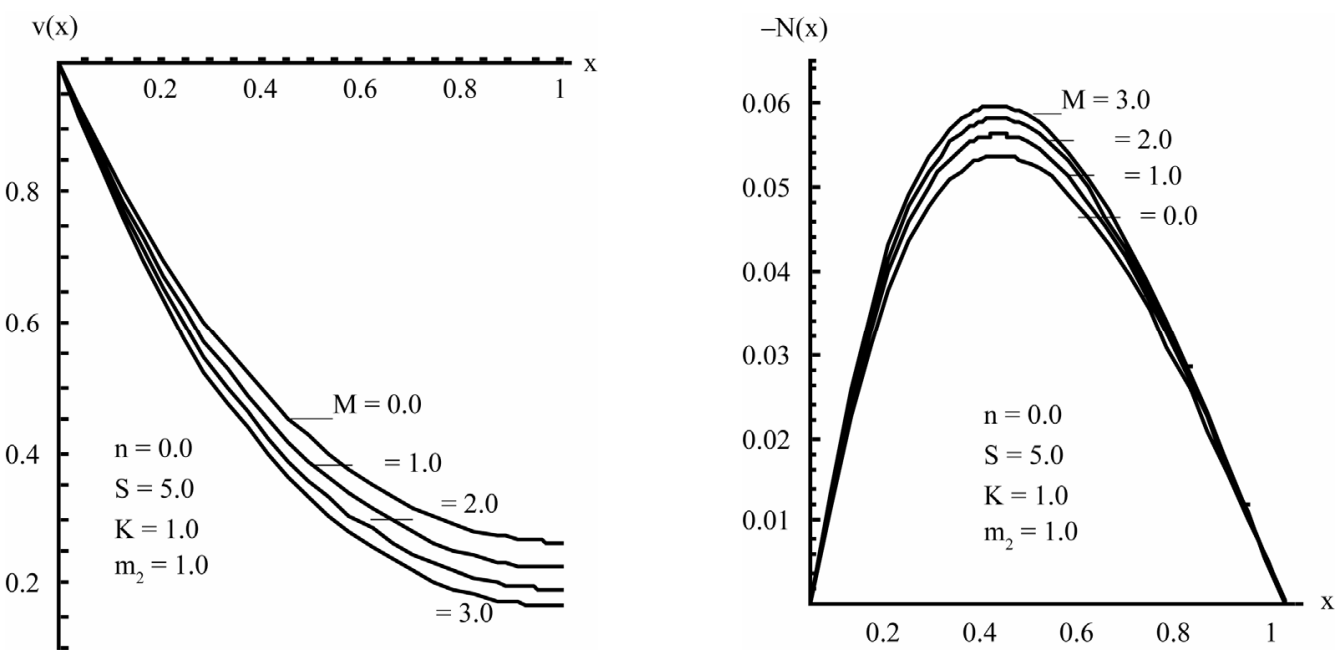

(2)

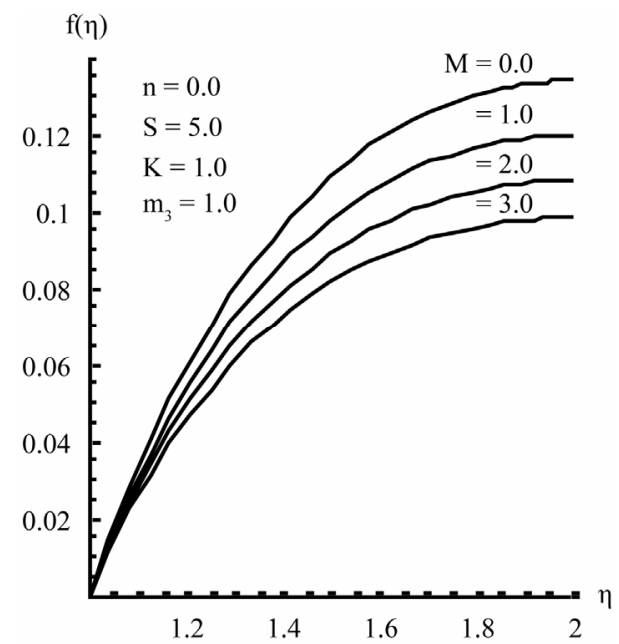

(a)

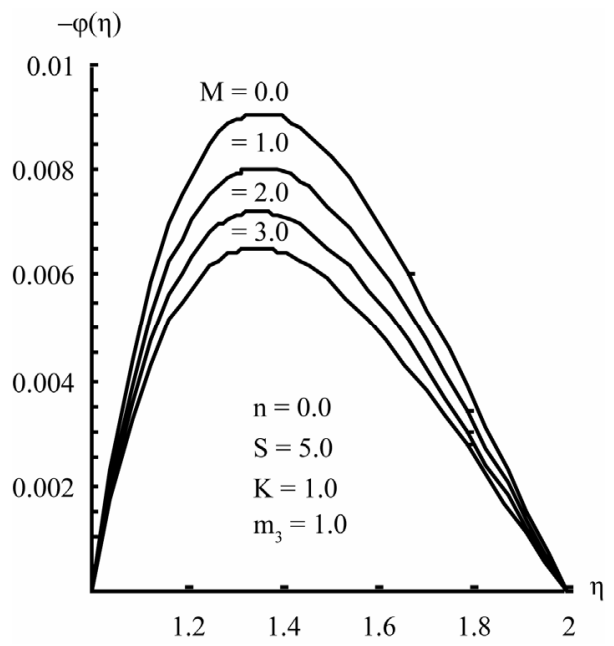

(b)

(3)

Figure 4. (a) velocity and (b) micro-rotation profiles for different values of $M$ for $n=0.0$. 

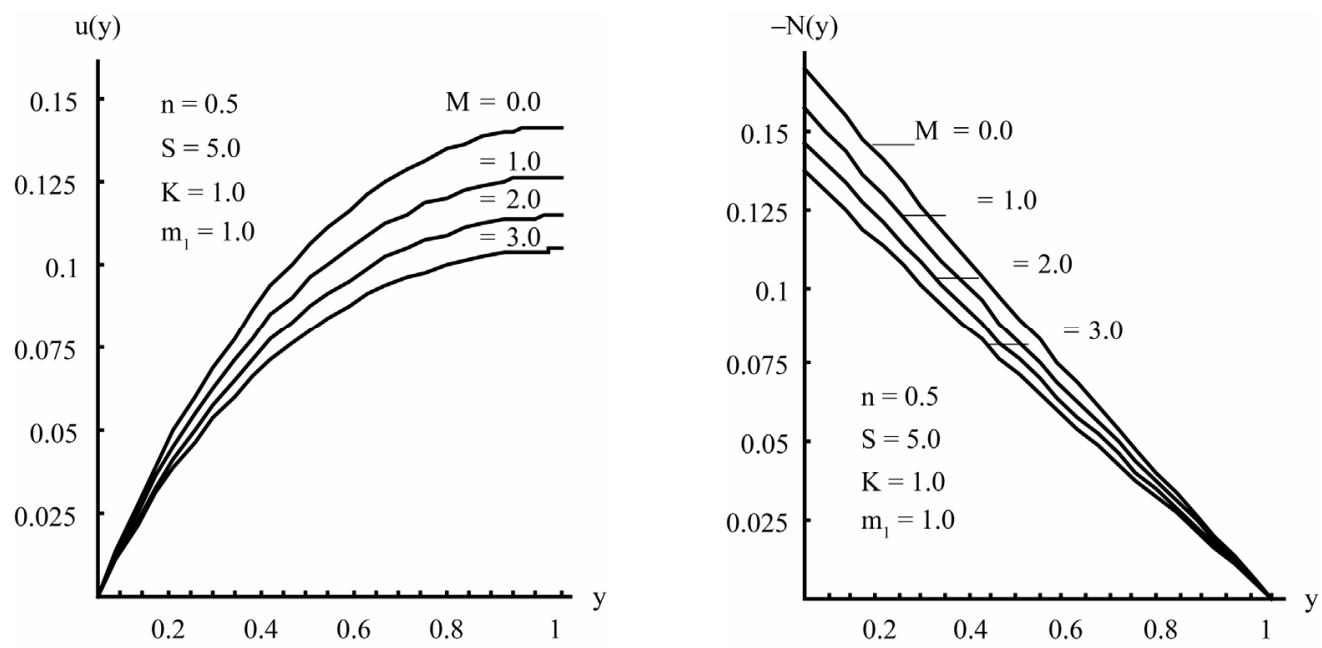

(1)
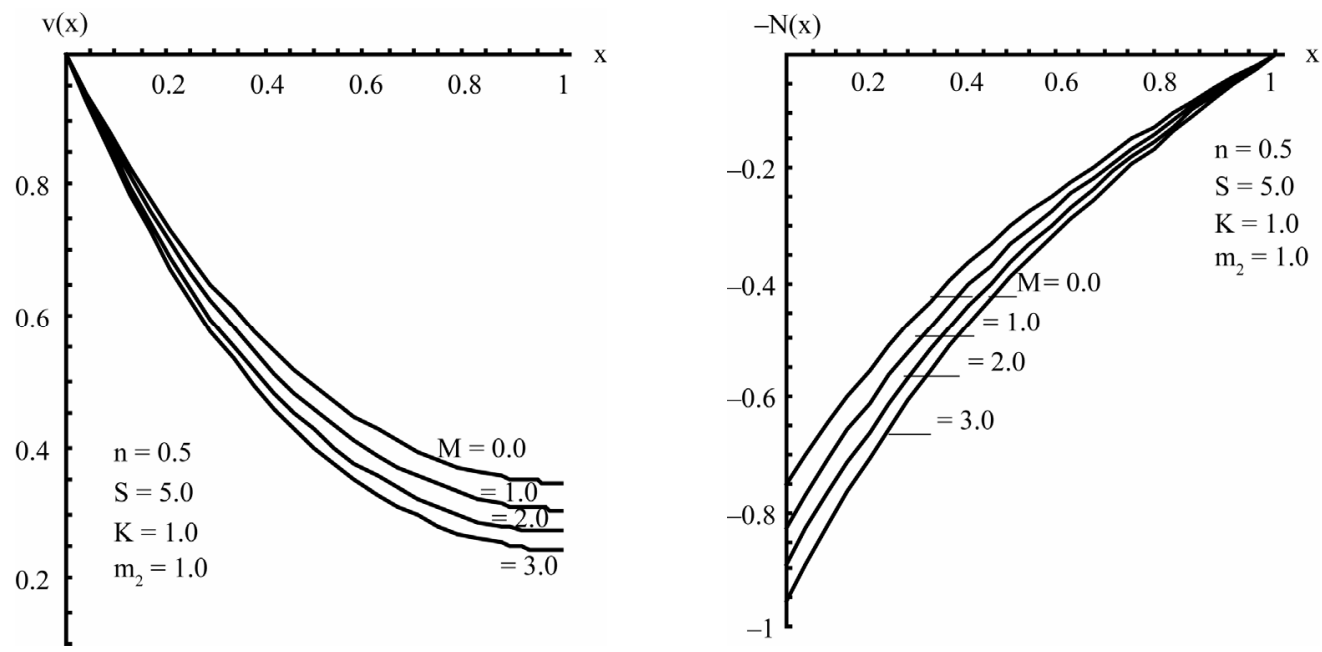

(2)
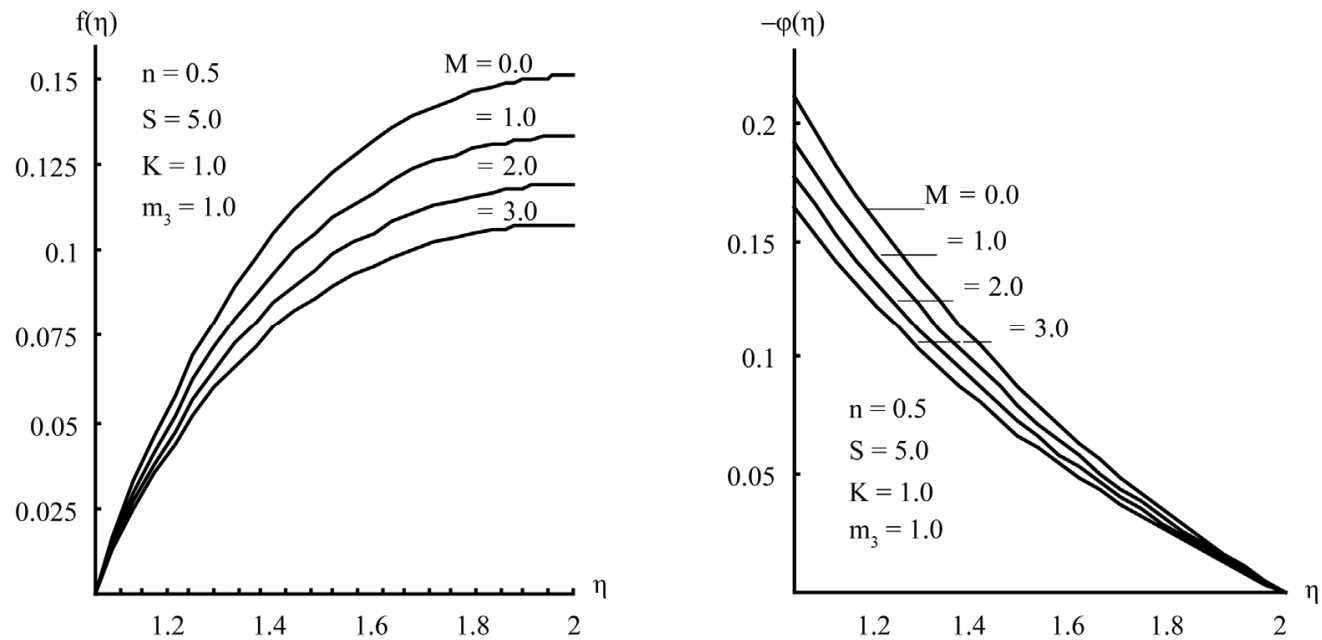

(a)

(b)

(3)

Figure 5. (a) velocity and (b) micro-rotation profiles for different values of $M$ for $n=0.5$. 

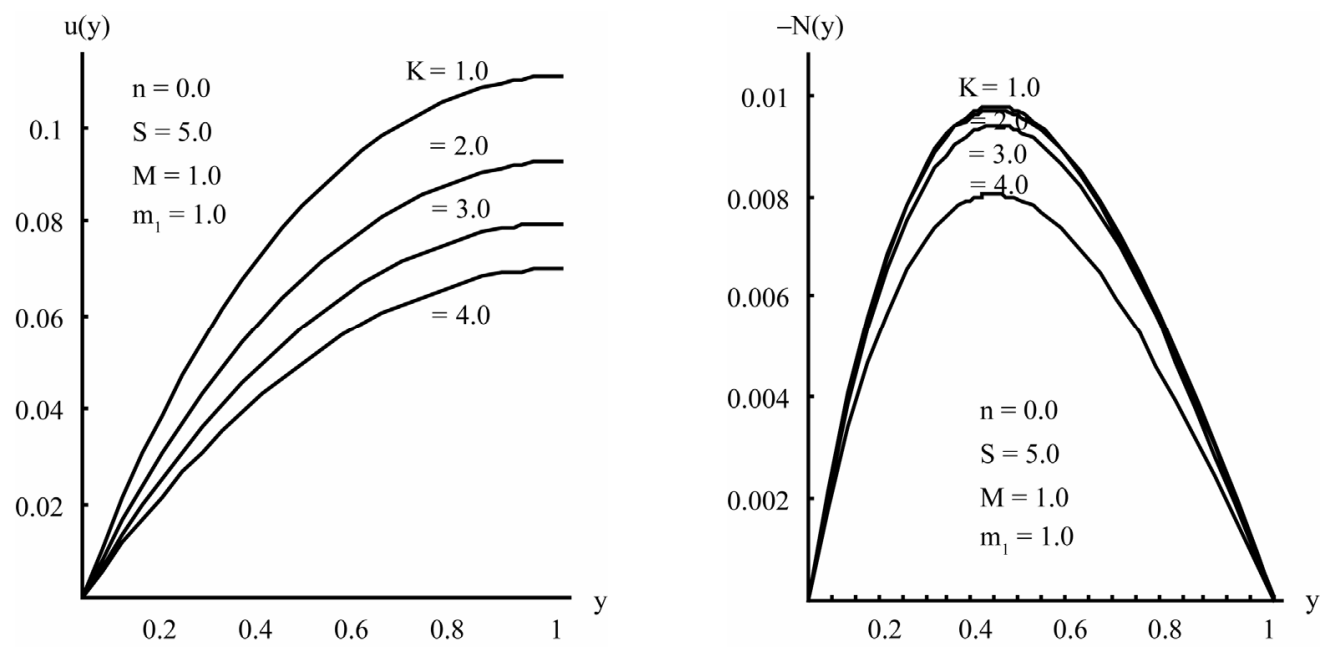

(1)
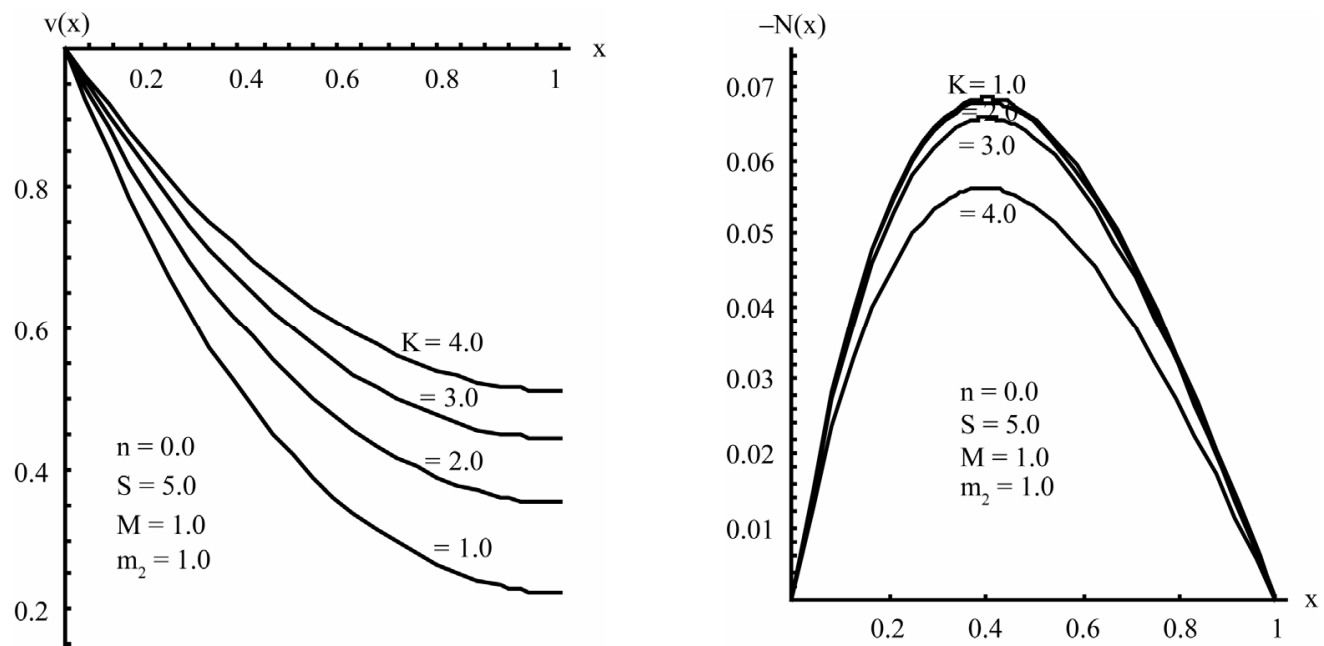

(2)

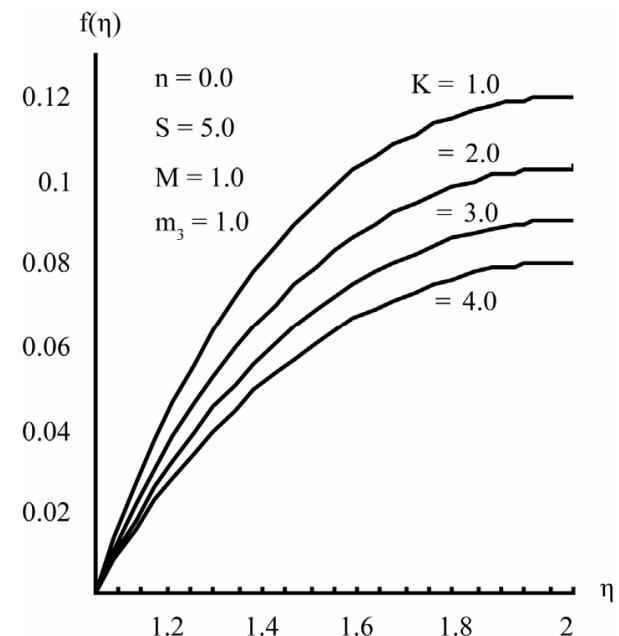

(a)

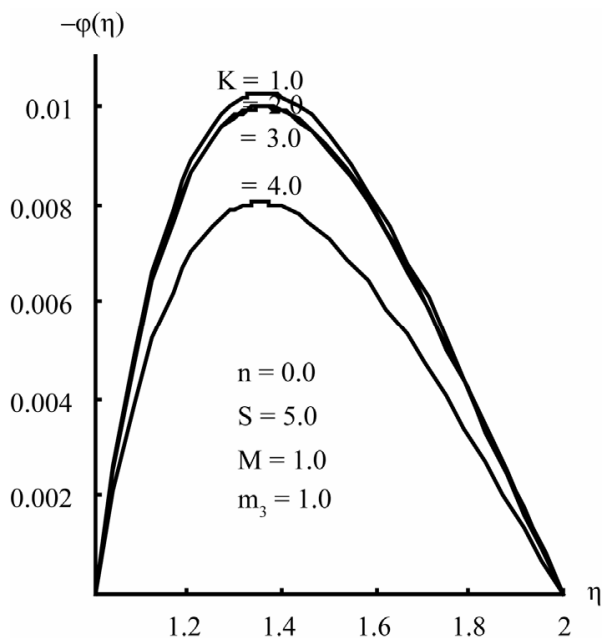

(b)

(3)

Figure 6. (a) velocity and (b) micro-rotation profiles for different values of $K$ for $n=0.0$. 

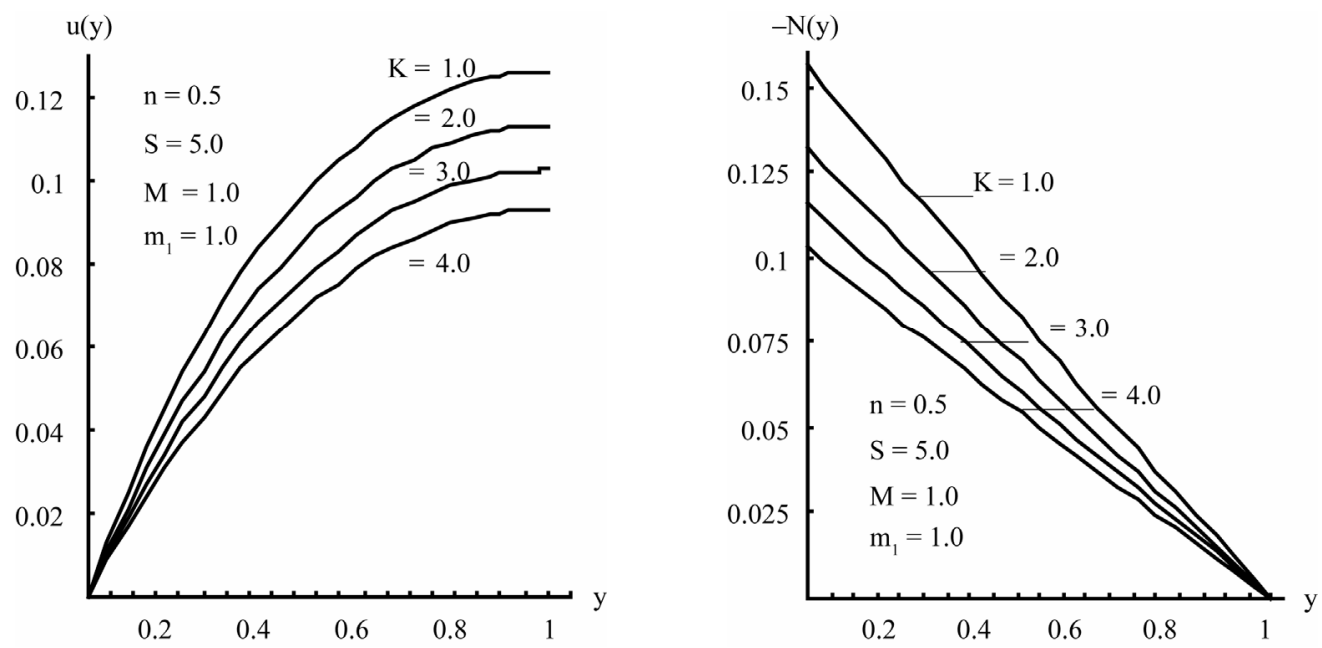

(1)
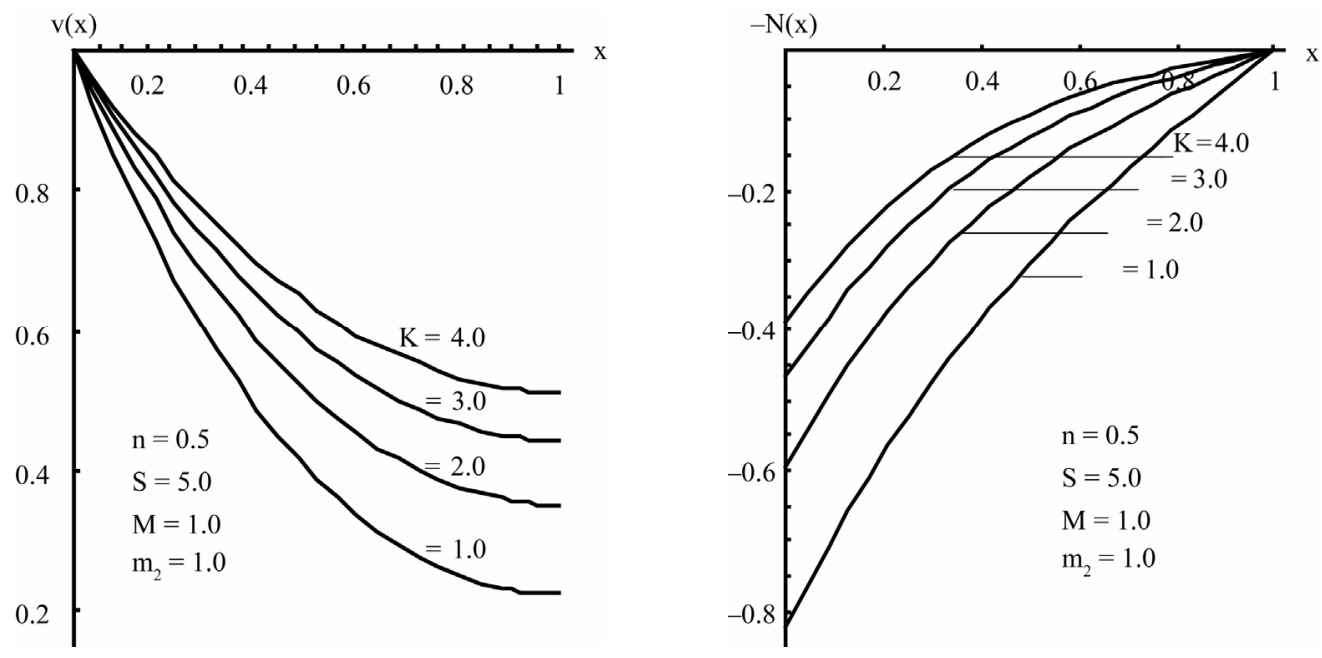

(2)
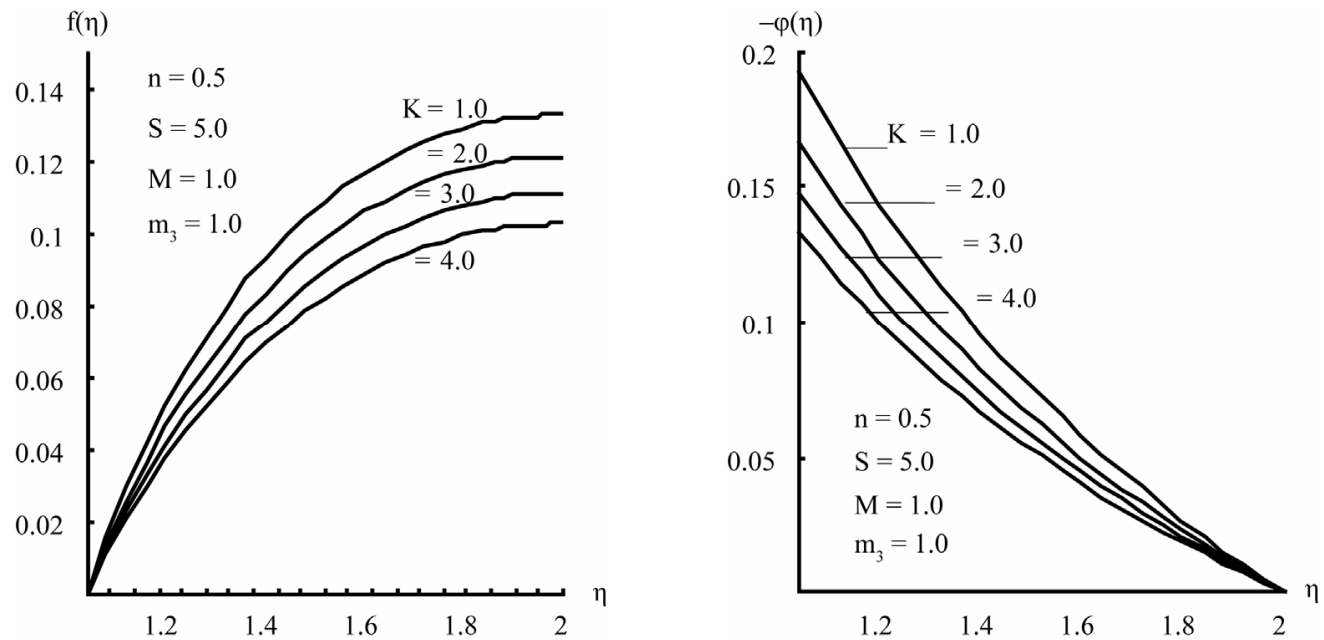

(a)

(b)

(3)

Figure 7. (a) velocity and (b) micro-rotation profiles for different values of $K$ for $n=0.5$. 

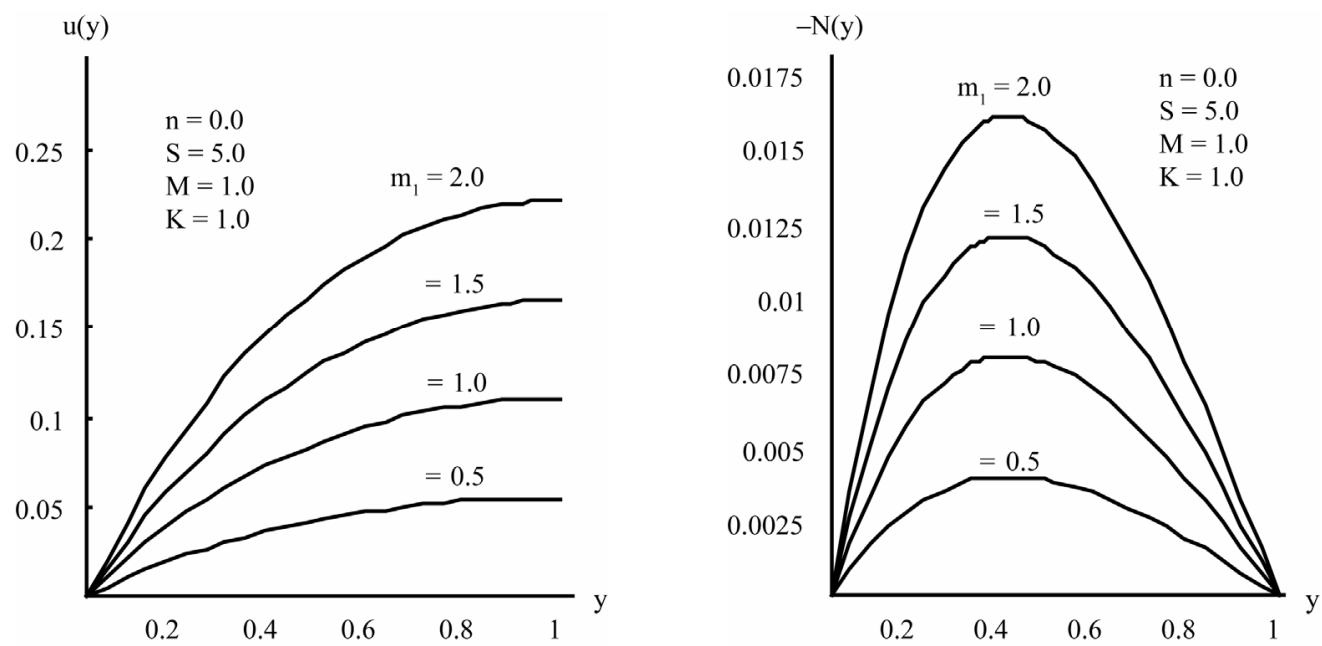

(1)
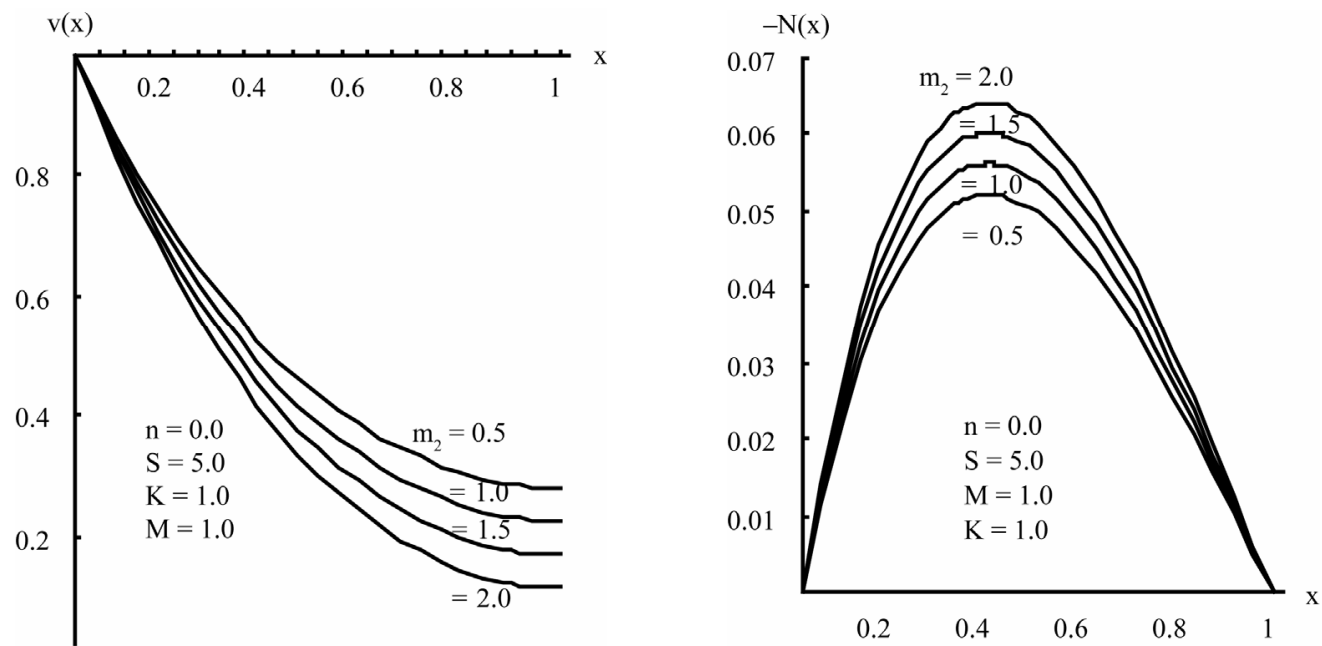

(2)
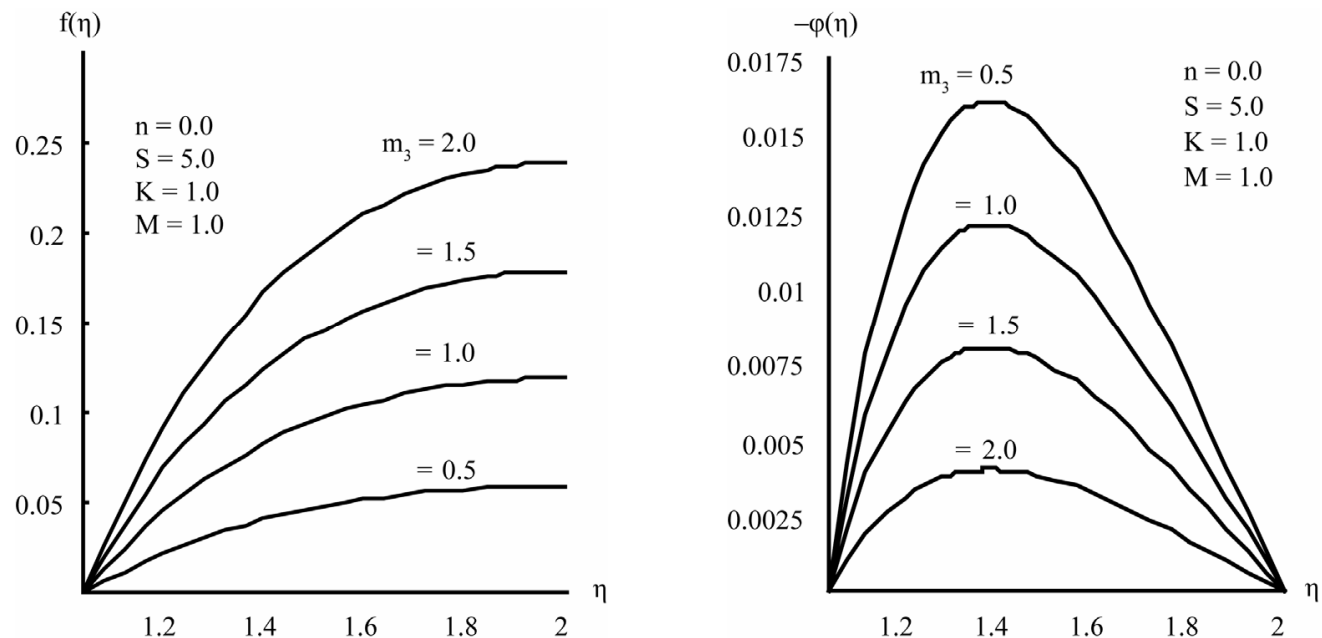

(a)

(b)

(3)

Figure 8. (a) velocity and (b) micro-rotation profiles for different values of $m_{1}, m_{2}$ and $m_{3}$ for $n=0.0$. 

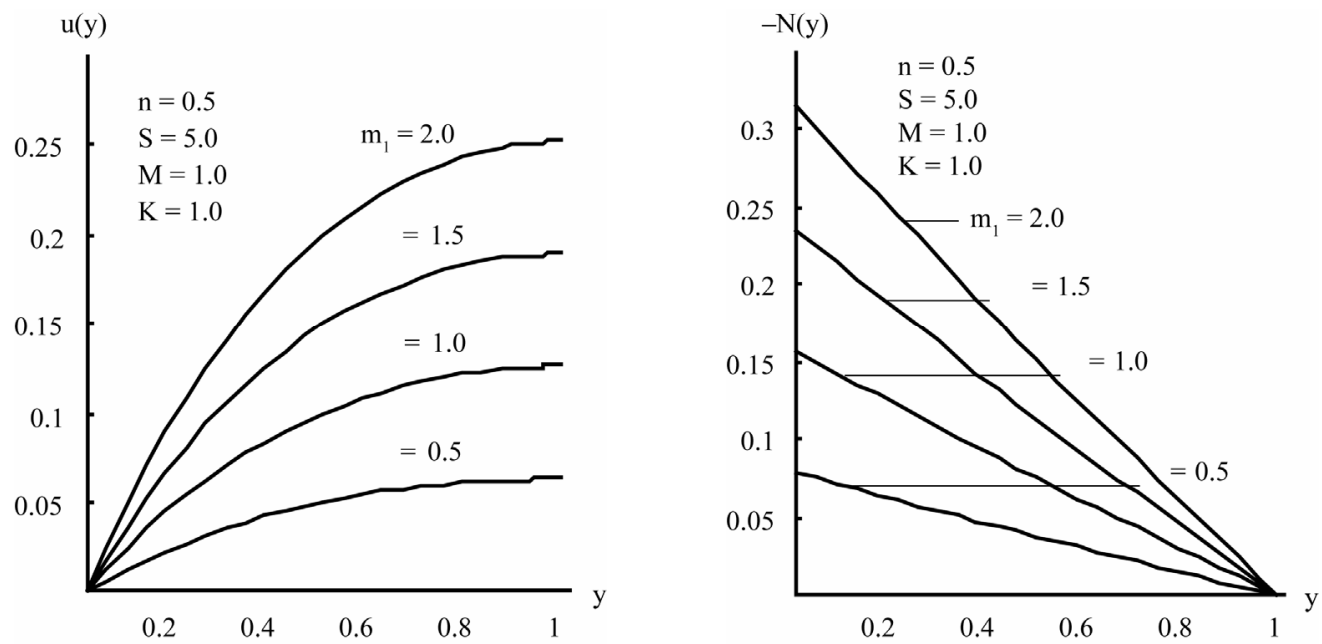

(1)
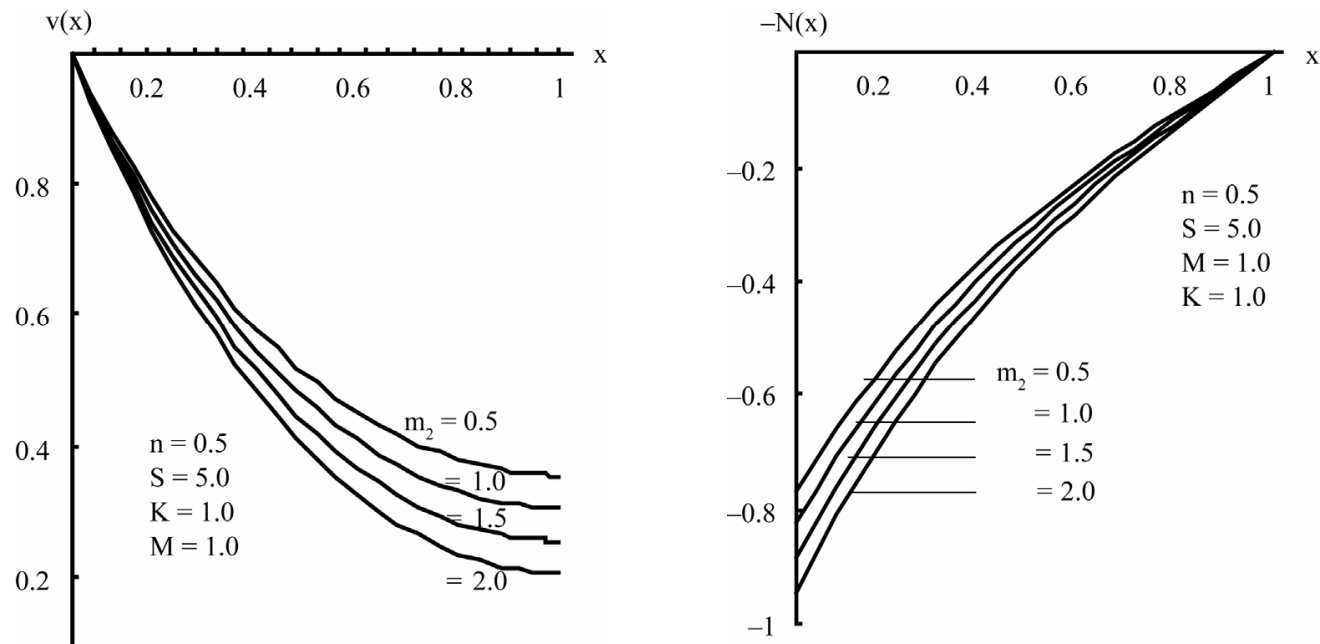

(2)

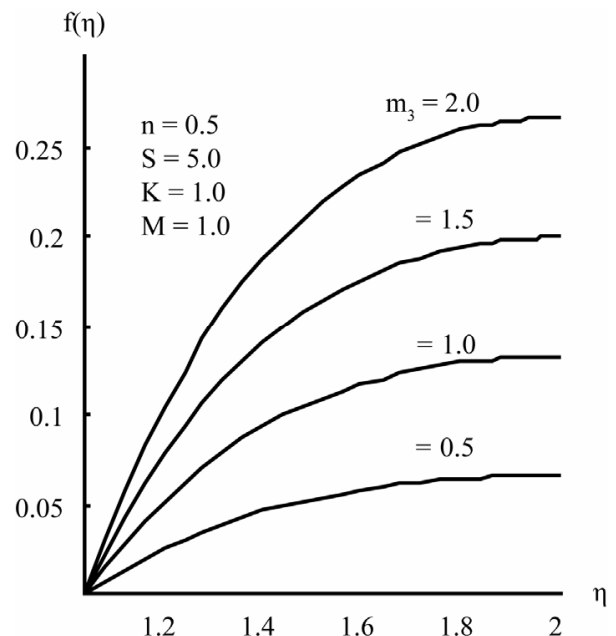

(a)

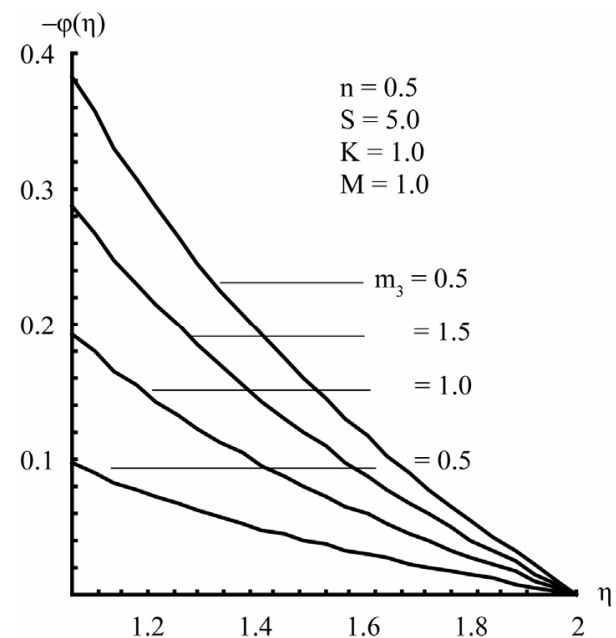

(b)

(3)

Figure 9. (a) velocity and (b) micro-rotation profiles for different values of $m_{1}, m_{2}$ and $m_{3}$ for $n=0.5$. 

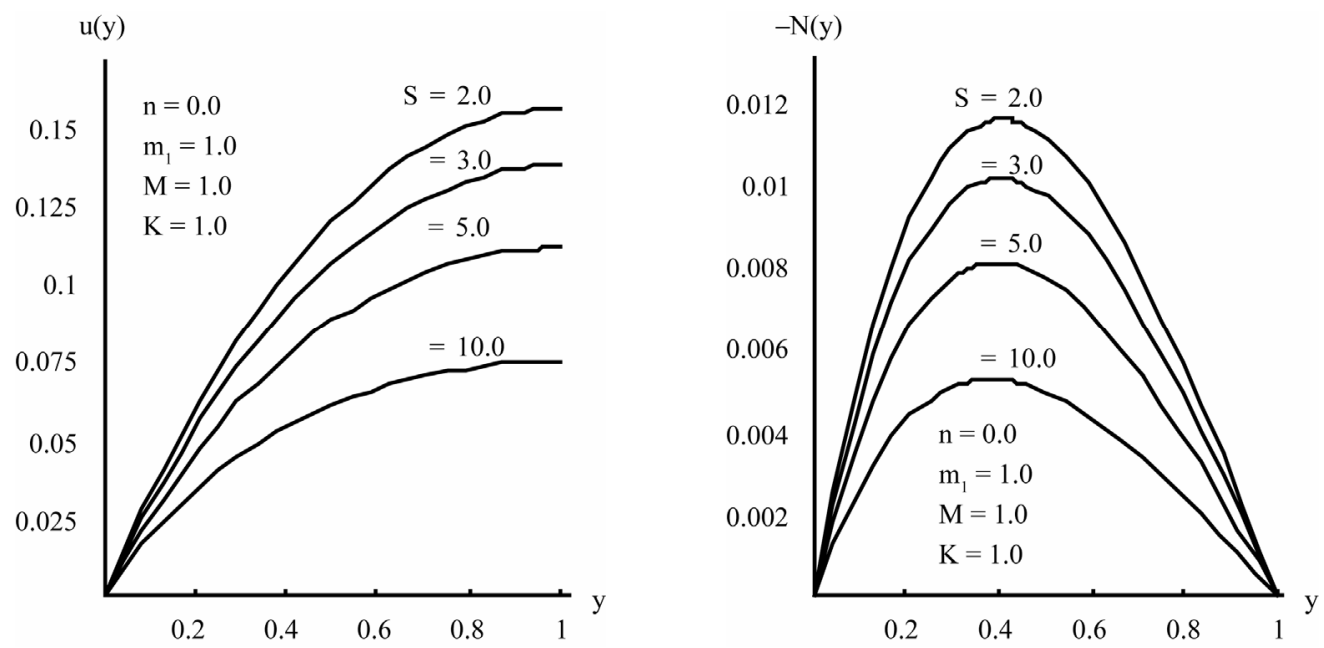

(1)
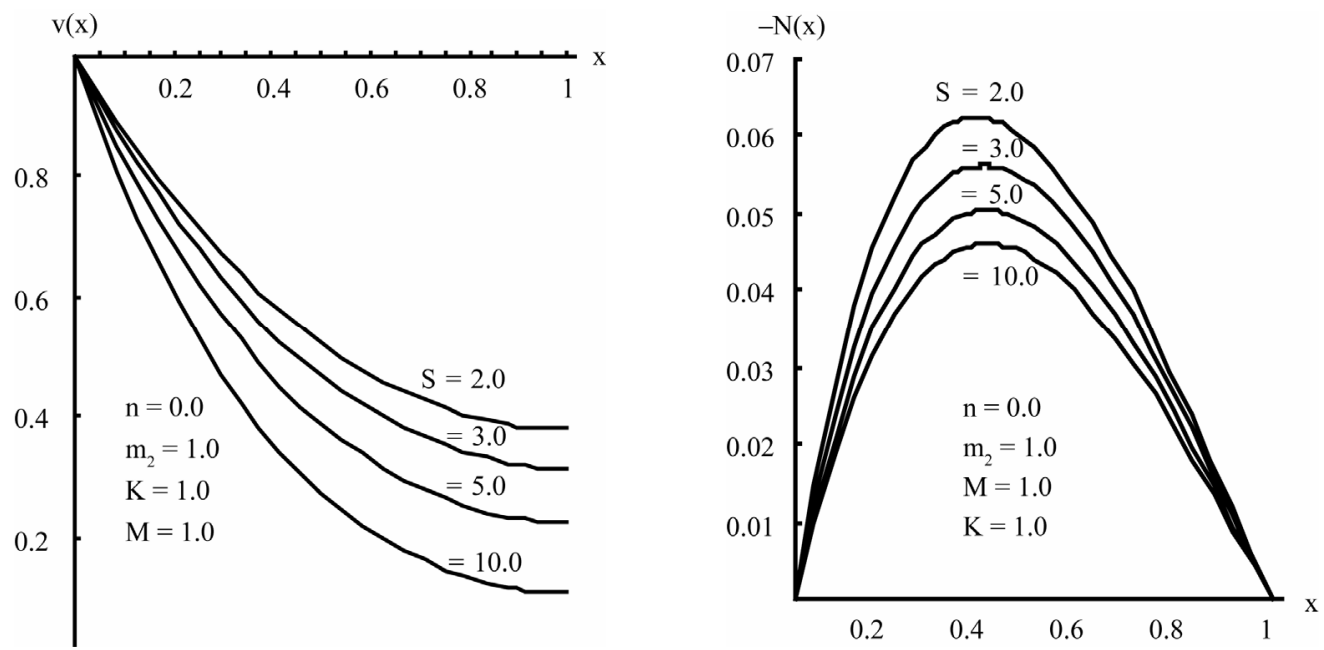

(2)
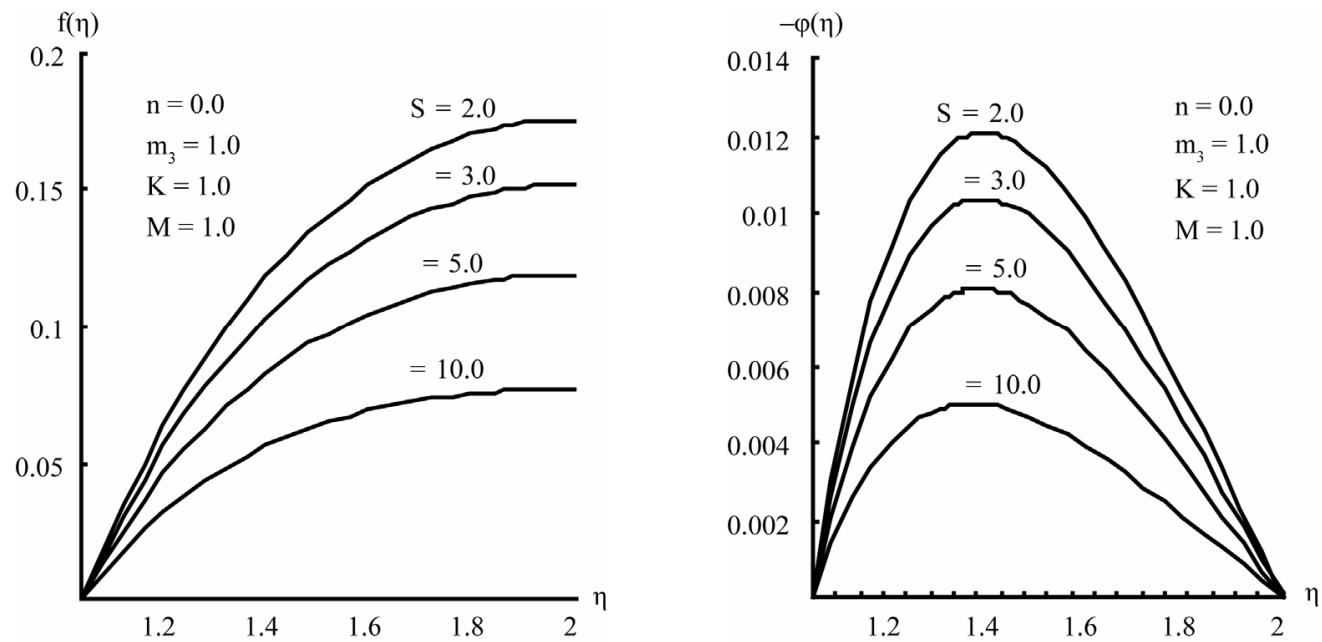

(a)

(b)

(3)

Figure 10. (a) velocity and (b) micro-rotation profiles for different values of $S$ for $n=0.0$. 

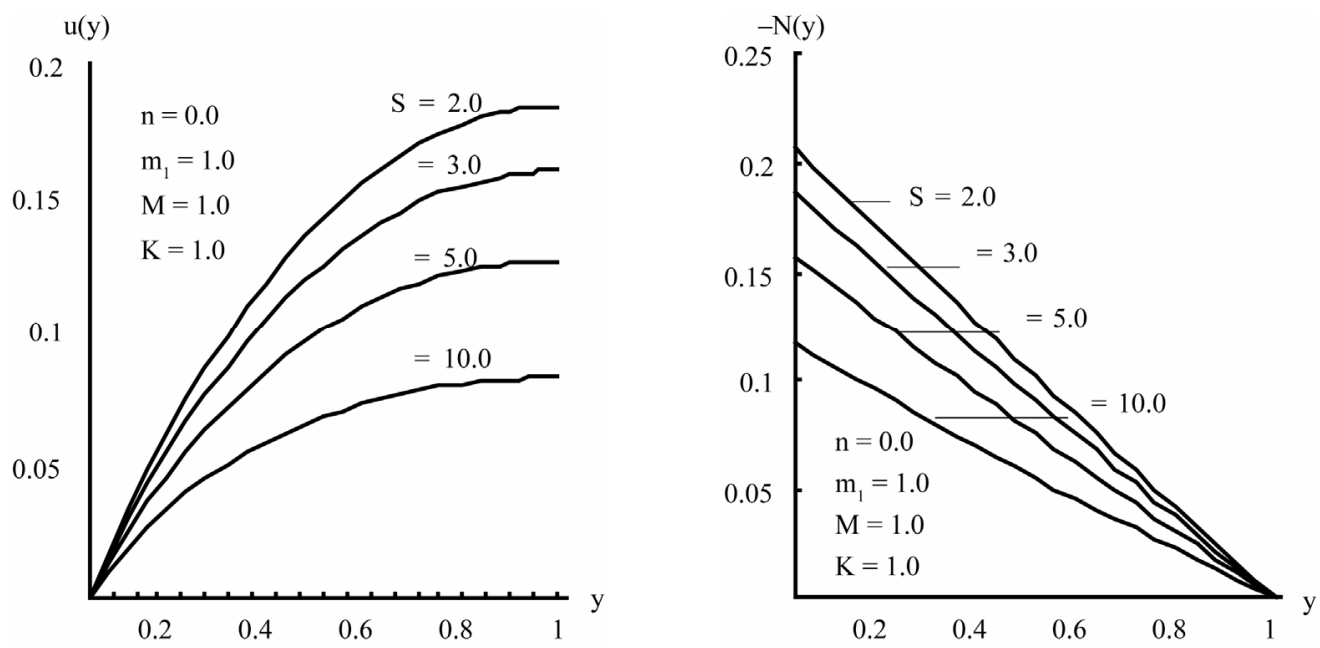

(1)
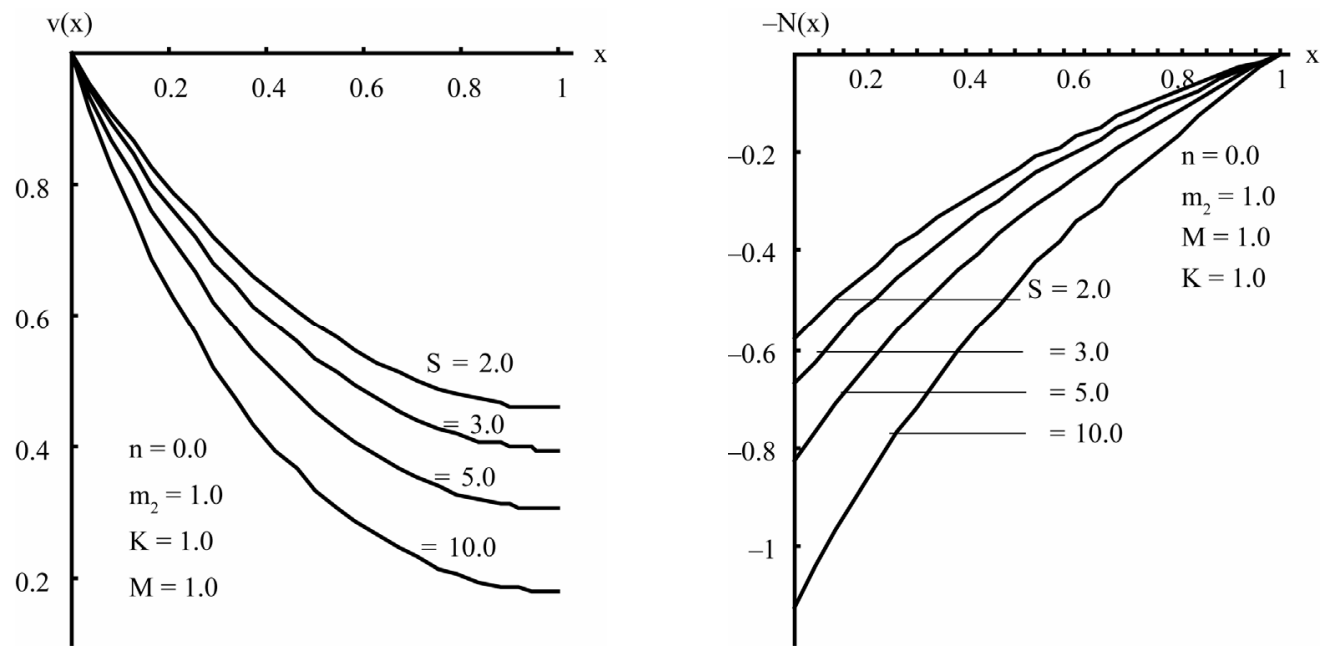

(2)
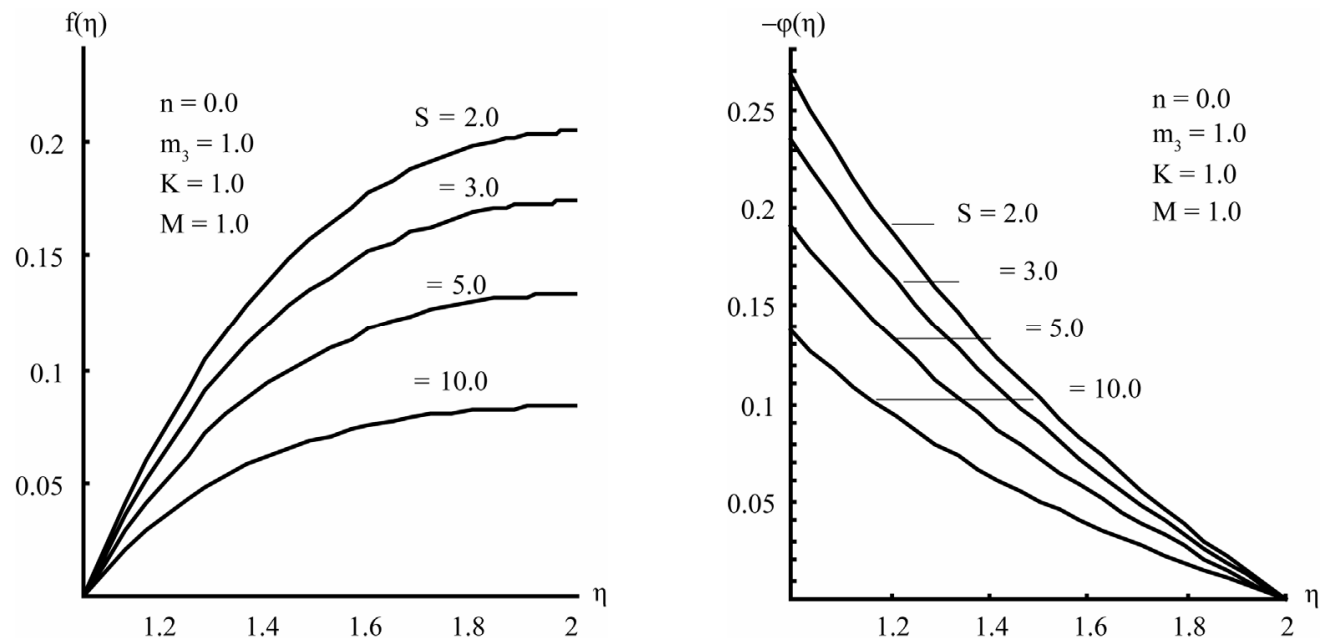

(a)

(b)

(3)

Figure 11. (a) velocity and (b) micro-rotation profiles for different values of $S$ for $n=0.5$. 
when compared with the case when there is no rotation at the boundary.

\section{References}

[1] W. C. Tan and T. Masuoka, "Stokes First Problem for a Second Grade Fluid in a Porous Half-Space with Heated Boundary," International Journal of Non-Linear Mechanics, Vol. 40, No. 4, 2005, pp. 515-522. doi:10.1016/j.ijnonlinmec.2004.07.016

[2] W.C. Tan and. T. Masuoka, "Stokes First Problem for an Oldroyd-B Fluid in a Porous Half-Space,” Physics of Fluids, Vol. 17, No. 2, 2005, pp. 3101-3107. doi:10.1063/1.1850409

[3] C. Fetecau and C. Fetecau, "Decay of a Potential Vortex in an Oldroyd-B Fluid," International Journal of Engineering Science, Vol. 43, No. 3, 2005, pp. 340-351. doi:10.1016/j.ijengsci.2004.08.013

[4] C. Fetecau and C. Fetecau, "Unsteady Flows of Oldroyd-B Fluids in a Channel of Rectangular CrossSection,” International Journal of Non-Linear Mechanics, Vol. 40, No. 9, 2005, pp. 1214-1219. doi:10.1016/j.ijnonlinmec.2005.05.005

[5] C. Fetecau and C. Fetecau, "Starting Solutions for Some Unsteady Unidirectional Flows of a Second Grade Fluid," International Journal of Engineering Science, Vol. 43, No. 10, 2005, pp. 781-789. doi:10.1016/j.ijengsci.2004.12.009

[6] T. Hayat, S. Nadeem, S. Asghar and A. M. Siddiqui, "Unsteady MHD Flow Due to Eccentrically Rotating Porous Disk and a Third Grade Fluid at Infinity,” International Journal of Applied Mechanics and Engineering, Vol. 11, No. 2, 2006, pp. 415-419.

[7] T. Hayat and A. H. Kara, "Couette Flow of a Third Grade Fluid with Variable Magnetic Field,” Mathematical and Computer Modelling, Vol. 43, No. 1-2, 2006, pp. 132-137. doi:10.1016/j.mcm.2004.12.009

[8] T. Hayat, S. B. Khan and M. Khan, "The Influence of Hall Current on the Rotating Oscillating Flows of an Oldoyd-B Fluid in Porous Medium," Nonlinear Dynamics, Vol. 47, No. 4, 2007, pp. 353- 362. doi:10.1007/s11071-006-9034-z

[9] C. I. Chen, C. K. Chen and Y. T. Yang, "Unsteady Unidirectional Flow of an Oldoyd-B Fluid in a Circular Duct with Different Given Volume Flow Rate Conditions,” Heat and Mass Transfer, Vol. 40, 2004, pp. 203-209. doi:10.1007/s00231-002-0350-7

[10] C. I. Chen, C. K. Chen and Y. T. Yang, "Unsteady Unidirectional Flow of a Second Grade Fluid between the Parallel Plates with Different Given Volume Flow Rate Conditions," Applied Mathematics and Computation, Vol. 137, No. 2, 2003, pp. 437-450. doi:10.1016/S0096-3003(02)00149-2

[11] A. C. Eringen, “Simple Microfluids," International Journal of Engineering Science, Vol. 2, No. 2, 1964, pp. 205-217. doi:10.1016/0020-7225(64)90005-9

[12] A. C. Eringen, “Theory of Micropolar Fluids,” Interna- tional Journal of Mathematics and Mechanics, Vol. 16, 1966, pp. 1-18.

[13] A. C. Eringen, “Theory of Micropolar Fluids,” Journal of Mathematical Analysis and Applications, Vol. 38, No. 2, 1972, pp. 480-496. doi:10.1016/0022-247X(72)90106-0

[14] A.C. Eringen, "Microcontinuum Field Theories. II: Fluent Media,” Springer, New York, 2001.

[15] Y. Y. Lok, P. Phang, N. Amin and I. Pop, "Unsteady Boundary Layer Flow of a Micropolar Fluid near the Forward Stagnation Point of a Plane Surface," International Journal of Engineering Science, Vol. 41, 2003, pp. 173-186. doi:10.1016/S0020-7225(02)00146-5

[16] R. Nazar, N. Amin, D. Filip and I. Pop, "Stagnation Point Flow of a Micropolar Fluid towards a Stretching Sheet," International Journal of Non-Linear Mechanics, Vol. 39, No. 7, 2004, pp. 1227-1235. doi:10.1016/j.ijnonlinmec.2003.08.007

[17] A. M. Siddiqui, R. Mahmood and Q. K. Ghori, "Homotopy Perturbation Method for Thin Film Flow of a Third Grade Fluid down an Inclined Plane," Chaos, Solitons and Fractals, Vol. 35, No. 1, 2008, pp. 140-147. doi:10.1016/j.chaos.2006.05.026

[18] A. Moncef, "Numerical Study for Micropolar Flow over a Stretching Sheet," Computational Materials Science, Vol. 38, No. 4, 2007, pp. 774-780. doi:10.1016/j.commatsci.2006.05.014

[19] A. M. Siddiqui, R. Ahmad and Q. K. Ghori, "Thin Film Flow of Non-Newtonian Fluid on a Moving Belt,” Chaos, Solitons and Fractals, Vol. 33, 2007, pp. 1006-1016. doi:10.1016/j.chaos.2006.01.101

[20] A. M. Siddiqui, R. Mahmood and Q. K. Ghori, "Homotopy Perturbation Method for Thin Film Flow of a Fourth Grade Fluid down a Vertical Cylinder,” Physical Letters $A$, Vol. 352, 2006, pp. 404-410. doi:10.1016/j.physleta.2005.12.033

[21] M. Sajid, N. Ali and T. Hayat, "On Exact Solutions for Thin Film Flows of a Micropolar Fluid," Communications in Nonlinear Science and Numerical Simulation, Vol. 14, No. 2, 2009, pp. 451-461. doi:10.1016/j.cnsns.2007.09.003

[22] M. Hameed and S. Nadeem, "Unsteady MHD Flow of a Non-Newtonian Fluid on a Porous Plate," Journal of Mathematical Analysis and Applications, Vol. 325, 2007, pp. 724-733. doi:10.1016/j.jmaa.2006.02.002

[23] G. M. Abdel-Rahman, "Studying Effect of MHD on Thin Films of a Micropolar Fluid,” Physica B, Vol. 404, No. 21, 2009, pp. 3859- 3866. doi:10.1016/j.physb.2009.07.112

[24] G. Lukaszewicz, "Micropolar Fluids: Theory and Applications,” Birkhauser, Basel, 1999.

[25] D. A. S. Ress and I. Pop, "Free Convection Boundary Layer Flow of a Micropolar Fluid from a Vertical Flat Plate,” IMA Journal of Applied Mathematics, Vol. 61, 2001, pp. 179-191. doi:10.1093/imamat/61.2.179

[26] G. Ahmadi, "Self-Similar Solution of Incompressible Micropolar Boundary Layer Flow over a Semi-Infinite Flat Plate," International Journal of Engineering Science, 
Vol. 14, No. 7, 1976, pp. 639-646. doi:10.1016/0020-7225(76)90006-9

[27] S. K. Jena and M. N. Mathur, "Similarity Solutions for Laminar Free Convection Flow of a Thermo-Micropolar Fluid Past a Non-Isothermal Flat Plate,” International Journal of Engineering Science, Vol. 19, No. 11, 1981, pp. 1431-1439. doi:10.1016/0020-7225(81)90040-9

[28] G. S. Guram and A. C. Smith, "Stagnation Flows of Mi- cropolar Fluids with Strong and Weak Interactions," Computers \& Mathematics with Applications, Vol. 6, No. 2, 1980, pp. 213-233. doi:10.1016/0898-1221(80)90030-9

[29] A. Ishak, R. Nazar and I. Pop, "Magnetohydrodynamic (MHD) Flow and Heat Transfer Due to a Stretching Cylinder," Energy Conversion and Management, Vol. 49, No. 11, 2008, pp. 3265-3269. doi:10.1016/j.enconman.2007.11.013 